\title{
Biomarcadores en monitoreo de exposición a metales pesados en metalurgia
}

\author{
Augusto V. Ramírez ${ }^{1}$ \\ En los trabajos de mina... los humos de sulfuro alcanzan rápidamente a los mineros y los matan. \\ Plinius Secundus, $77 D C$.
}

Resumen

Palabras clave
La salud del trabajador es el hecho más importante en la vigilancia por exposición a tóxicos industriales. En metalurgia extractiva, existe exposición a muchos elementos y compuestos químicos. El avance tecnológico actual permite realizar mediciones muy precisas en medios biológicos. Monitoreo biológico es medir la concentración del agente tóxico en los medios biológicos del trabajador, para establecer niveles de exposición y medidas de control en el ambiente laboral. Un indicador biológico de exposición valora la cantidad absorbida de un químico o de los subproductos de su biotransformación en medios biológicos, lo que permite cuantificar al agente en el organismo. En la exposición industrial para valorar daño renal, usamos función renal, citotoxicidad específica, proteínas de peso molecular bajo o enzimas excretadas. Para el sistema nervioso, se desarrolla indicadores 'substitutos'y, en genotoxicidad, aductos del ADN. El presente trabajo revisa indicadores de exposición para metales en metalurgia extractiva.

Metales tóxicos; metalurgia; exposición a riesgos ambientales; monitoreo del ambiente.

\section{Biomarkers used to monitor heavy metal exposure in metallurgy}

\section{Abstract}

Worker's health is the most important fact in heavymetal exposition surveillance. In extractive metallurgy there is exposition to many elements and chemical compounds. Current laboratory technological advances allow us to develop biologic media's measurements for use in occupational health assessment.

Monitoring is measuring, assessing and interpreting biological media and environment parameters, to prevent and avoid health risks. It implies establishing control measures in labor environment defining exposition levels not causing adverse effects on workers.

An exposition's biological indicator doses blood metal or byproducts biotransformation into biological media in order to quantify body chemistry. In kidney toxicity, we use function and citotoxicity indicators, low molecular weight proteins and urinary enzymes. In neurological toxicity, substitute indicators are being developed. And in genotoxicity, DNA adducts. This paper revises indicators for main heavy metals in extractive metallurgy.

Keyword: Toxic metals; metallurgy; environmental exposure; environmental monitoring.

1 American College of Occupational and Environment Medicine. Lima, Perú.

\section{INTRODUCCIÓN}

La preocupación por la salud del trabajador data de antiguo, pero recién con el desarrollo de la máquina de vapor, que marca el inicio de la segunda revolución industrial, se desarrollan acciones tendientes a protegerlo de condiciones higiénicas adversas. En los últimos 50 años, con la aparición de nuevas sustancias químicas, si bien es verdad punto de apoyo en el avance industrial, el panorama se complica $y$ no es menos cierto que este avance ha producido empeoramiento de las condiciones de higiene y salud en el trabajo, que literalmente agreden al trabajador.

Es pues imprescindible conocer la naturaleza y acción biológica de cada agente, para controlarlo con exámenes médico ocupacionales de exposición. El uso de biomarcadores en exposición a metales pesados tiene una finalidad práctica: cuidar 
la salud en el trabajo, manejando índices de exposición, de efecto y de susceptibilidad, válidos para la toma de acciones correctivas de salud laboral y minimizar o suprimir el efecto del tóxico. En salud ocupacional, la monitorización de la exposición es el procedimiento rutinario de medición, valoración e interpretación de parámetros biológicos y ambientales, con el fin de detectar en forma precoz los posibles riesgos para la salud en el trabajo. Así se ha venido aplicando desde hace más de 4 décadas. Sin embargo, la legislación peruana actual omite establecer indicadores biológicos de exposición, lo que crea un vacío en la valoración de la exposición laboral a metales pesados, sobre todo sabiendo que solo un examen médico ocupacional que identifique antecedentes o estigmas de exposición y se acompañe de valoración de indicadores biológicos, es el único medio válido en la vigilancia de salud laboral $\left({ }^{1}\right)$.

\section{MONITOREO BIOLÓGICO Y BIOMARCADORES}

Monitorización es el procedimiento de medición e interpretación de parámetros biológicos y ambientales, mientras que monitoreo biológico es el procedimiento de salud ocupacional por el cual se mide un tóxico potencial, sus metabolitos o un efecto químico no deseado en una muestra biológica, con el propósito de evaluar la exposición a ese agente. La exposición puede ser valorada midiendo la concentración del tóxico en muestras de aire (monitorización ambiental) o identificando parámetros biológicos en el trabajador: sangre, orina o aire exhalado (monitorización biológica). A estos últimos se les llama indicadores biológicos de exposición o biomarcadores. El monitoreo biológico mide la cantidad de agente absorbido, independiente de la vía de ingreso, y es una actividad regular $y$ repetitiva con fines preventivos, por lo que no debe ser confundida en clínica con los llamados procedimientos diagnósticos.

El monitoreo biológico presupone establecer cuatro condiciones:

1. Que la sustancia química o sus metabolitos (biomarcador) estén presentes en algún tejido, excreción o fluido corporal y que además tenga un nivel apropiado para su medición.

2. Que los métodos de análisis de la muestra sean válidos, prácticos y que además estén disponibles en el medio.

3. Que la estrategia de medición sea adecuada, es decir que la muestra sea representativa y tomada en el tiempo indicado.

4. Que los resultados puedan ser interpretados matemáticamente y que sean estadísticamente significativos.

Biomarcador, entonces, es una sustancia química, generalmente un tóxico, o los metabolitos que resulten de su biotransformación o cualquier alteración bioquímica precoz, cuya determinación en los líquidos biológicos, tejidos o aire exhalado permita evaluar la intensidad de exposición o riesgo para la salud. Ergo, un biomarcador puede ser usado con variada finalidad, dependiendo del propósito del estudio y del tipo de exposición. Por otro lado, en clínica, los biomarcadores nos ayudan a evaluar el riesgo de salud, a verificar relación causaefecto y dosis-efecto y, particularmente en salud ocupacional, a controlar riesgos. Por estas características, un indicador biológico puede ser usado en salud general (mide la efectividad de un tratamiento o ayuda con el pronóstico) y en salud pública u ocupacional, al medir exposición $\left(^{2}\right)$.

\section{CLASIFICACIÓN DE LOS BIOMARCADORES}

Existe una gran variedad de biomarcadores utilizados para valorar 
eventos biológicos en exposición a tóxicos industriales. Veamos los principales.

\section{Biomarcadores de exposición o de dosis interna}

Un paso crucial en el proceso de valoración de riesgo de salud es demostrar la exposición del trabajador a substancias riesgosas. ¿Cómo? Pues, demostrando evidencia de carga corporal del tóxico proveniente de la exposición. Un biomarcador de exposición valora la dosis interna determinando el agente o subproductos de biotransformación en medios biológicos (Figura 1), permitiendo su cuantificación en el organismo. Por ejemplo, plomo sanguíneo $(\mathrm{Pb}-\mathrm{S})\left({ }^{3}\right)$.

\section{Biomarcadores de efecto}

Un biomarcador de efecto es el parámetro biológico que refleja la interacción del químico con los receptores biológicos. Como estas alteraciones anteceden al daño estructural, su detección permite la identificación precoz de exposiciones excesivas o peligrosas y tomar medidas preventivas; ej., la carboxihemoglobina es un biomarcador de efecto, además de tener correlación significativa con exposición ambiental a monóxido de carbono y de reflejar dosis interna unida al tejido blanco (target).

La inhibición de la dehidratasa del ácido delta amino levulínico (ALA-D) en hematíes es altamente sensible en la exposición a

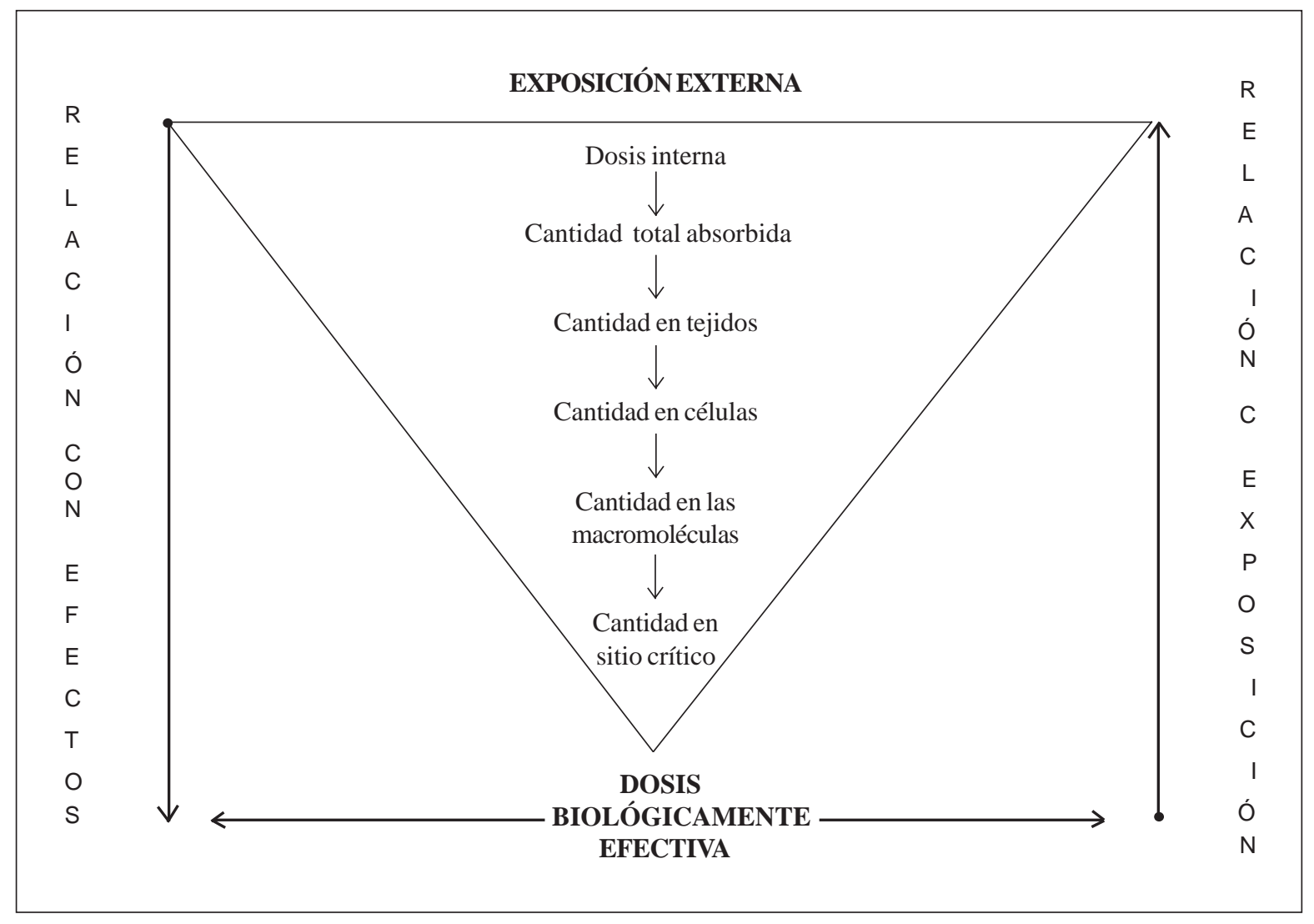

Figura 1. Progresión de la acción de los biomarcadores de dosis interna hasta el nivel molecular de dosis efectiva. Modificado de WHO, IPCS Environmental Health Criteria 155 Biomarkers and Risk Assessment Geneva. 1993. 
plomo. Así, con $\mathrm{Pb}-\mathrm{S}$ entre 5 y $40 \mathrm{ug} / \mathrm{dL}$, ya es posible observar correlación negativa entre ALA-D y $\mathrm{Pb}-\mathrm{S}$. De ahí que, la ALA$D$ sea un indicador de efecto adecuado en la exposición a bajas concentraciones de $\mathrm{Pb}$, medio ambientales por ej.; por eso también es factible usarlo como indicador de exposición ocupacional a $\mathrm{Pb}\left(^{3}\right)$.

\section{Biomarcadores por órgano blanco}

\section{a. Biomarcadores de nefrotoxicidad}

El uso de biomarcadores de nefrotoxicidad data de los años 50, cuando se describe la intoxicación por cadmio en Japón. Pero, para usarlos como biomarcadores en exposición a plomo o mercurio, la evidencia todavía es insuficiente. Aún así, en salud ocupacional usamos los siguientes biomarcadores para daño renal:

- Funcionales: creatinina sérica y beta $_{2}-$ microglobulina

- Proteínas: albúmina, transferrina, globulina ligada a retinol.

- Marcadores de citotoxicidad, antígenos tubulares

- Enzimas urinarias: glutation-S-transferasa, NAG, beta-galactosidasa $\left({ }^{4}\right)$.

\section{b. Biomarcadores de hepatotoxicidad}

La hepatotoxicidad es causada por tóxicos que al ser metabolizados por el sistema citocromo P-450 alteran sus delicados sistemas enzimáticos. Por ejemplo, el efecto de algunos metales pesados sobre el hígado ha sido valorado por el daño a varias enzimas: aminotransferasas, dehidrogenasas, glutation-S-transferasas.

\section{c. Biomarcadores de neurotoxicidad}

Las investigaciones recomiendan abordar el problema bajo los siguientes aspectos:

- Aspecto neurofisiológico: Electroencefalograma, estudios de conducción nerviosa y potenciales evocados, son útiles en el diagnóstico de desórdenes neurológicos, pero no hay evidencia para usarlos en la identificación de efectos neurotóxicos iniciales y por tanto sirven poco en prevención.

- Aspecto neurocomportacional: Los métodos para evaluar alteración de la función cognitiva, aprendizaje y memoria son utilizados en exposición a solventes orgánicos y metales pesados. Pero, como indicadores de exposición a neurotóxicos, su uso es reciente y limitado.

- Aspecto neuroquímico: En las enfermedades causadas por neurotóxicos, el evento bioquímico precede a las alteraciones estructurales y a daños permanentes en el sistema nervioso central (SNC), dándonos información útil sobre efectos precoces y mensurables al inicio de la intoxicación. En general, todos los eventos bioquímicos que ocurren en el sistema nervioso son biomarcadores potenciales, pero su principal limitación es la inaccesibilidad del tejido nervioso.

\section{Indicadores sustitutos}

Vistas estas limitaciones, para evaluar riesgo de salud se ha desarrollado nuevas tecnologías en biomarcadores usando tejidos o medios biológicos accesibles. Se les llama indicadores 'substitutos del tejido nervioso' y destacan líquido cefalorraquídeo, sangre, plasma y células sanguíneas; enzimas, como acetil colinesterasa eritrocitaria, estearasa neurotóxica en linfocitos, MAO tipo B en plaquetas; calcio intracelular o fosfoinositol en plaquetas y linfocitos.

En neurotoxicidad, dosar 'indicadores sustitutos' nos permite evaluar con métodos accesibles alteraciones bioquímicas e identificar en forma precoz exposiciones excesivas para prevenir la aparición de daño irreversible. El criterio actual para usar biomarcadores en exposición a neurotóxicos 
recomienda su aplicación ya desde niveles bajos de exposición $\left({ }^{6}\right)$.

\section{d. Biomarcadores de genotoxicidad}

Se ha desarrollado técnicas para detectar gran variedad de carcinógenos: por ej., las aflatoxinas y las aminas aromáticas ya son usados como biomarcadores.

\section{Aductos}

Aductos son neomoléculas resultantes de la interacción y unión del xenobiótico o sus metabolitos con la hemoglobina o albúmina en el sitio target del químico. Los aductos, al estar constituidos por el propio agente ligado en el lugar de acción, pueden ser usados como biomarcadores de exposición. Así, los biomarcadores de susceptibilidad como respuesta a un agente químico reflejan la acción de factores internos o adquiridos que son independientes del nivel de exposición y sirven para indicarnos qué factores pueden contribuir a variar en mayor o menor grado la respuesta del organismo.

Con esta amplia variedad de biomarcadores, el trabajador no debe ser más un 'aparato detector ambulatorio de muestreo', y la sola idea de basar un programa de prevención para un tóxico midiendo su absorción en el organismo del trabajador, es anética $\left({ }^{7}\right)$.

\section{FUENTES DE ERROR EN LA TOMA DE LA MUESTRA}

Los errores en el uso de biomarcadores son producidos antes, durante o después del monitoreo. Sin embargo, los errores más frecuentes están dados por factores fisiológicos, toxicocinéticos, medio ambientales y no pocas veces contaminación durante colección de muestra. Visto así el problema, el laboratorio que analiza poco puede hacer para evitar o descubrir estos errores, que más bien deben ser manejados y resueltos por el personal de salud a cargo de la prueba.

Entre los principales errores en la toma de muestra tenemos:

Variación circadiana. Las concentraciones de muchos agentes químicos endógenos y varios exógenos tienen variación circadiana en los líquidos corporales. que es independiente de su ingesta.

Volumen urinario. El volumen de orina excretado depende del estado del hidratación del organismo y, como sabemos, el volumen influye en la concentración del agente en la orina.

Medio ambiente. Los contaminantes del medio ambiente, agua o aire, exponen al ser humano a una gran cantidad de tóxicos, como los metales pesados cadmio, plomo, mercurio y otros; por lo que se hace indispensable considerar probables exposiciones medioambientales.

Dieta y tabaco. Existe relación directa entre el tóxico ingerido con la dieta y su concentración en orina. Independiente de una fuente laboral, las vías más frecuentes de ingreso de un tóxico las encontramos en las bebidas y alimentos, que exponen al ser humano a una gran cantidad de tóxicos, como metales pesados -cadmio, plomo, mercurio, químicos orgánicos, DDT, dioxinas, PCBs, etc. El humo del tabaco contiene, entre muchos otros tóxicos, benceno, monóxido de carbono, HCN y cadmio.

Fisiología individual y hora de toma de muestra. Los cambios fisiológicos suceden día y noche y hay que tenerlos en cuenta al indicar un horario para colectar la muestra. La hora debe ser estandarizada y circunscrita a las primeras horas matinales.

Otras fuentes de error en la toma de muestra. Por otro lado, si analizamos el agente en sangre, la absorción cutánea durante el pinchazo puede causar un error sustancial en 
su interpretación. Además la sangre obtenida de una vena no representa necesariamente la sangre de todo el organismo, sino más bien su concentración local en la región donde se toma la muestra $\left({ }^{8}\right)$.

\section{ERRORES EN EL ALMACENAMIENTO DE LA MUESTRA}

Los factores de error en el almacenamiento de la muestra incluyen:

Evaporación: Este error se presenta principalmente cuando se monitorea solventes. La acidificación de la orina disminuye estas pérdidas.

Digestión química: Es un problema típico de los compuestos orgánicos

Precipitación y adsorción: La orina se comporta a menudo como una solución sobresaturada de ácido úrico o fosfatos que al ser almacenada tiende a precipitar. La adsorción en la superficie del recipiente es de menor importancia para orina que para sangre.

Contaminación: La contaminación puede provenir del ambiente de trabajo, sitio de toma de muestra, piel o ropa del trabajador, recipientes usados, aditivos, reactivos o del equipo analizador. Las tapas de caucho de los vacutainers de uso general son una fuente notoria de cadmio y otros elementos $\left({ }^{8}\right)$.

\section{INTERPRETACIÓN DE LOS RESULTADOS}

Los resultados del monitoreo biológico han de ser comparados con valores de referencia (VR) establecidos y aceptados internacionalmente. De hecho, puede existir discrepancias entre el VR de uso clínico y ocupacional, debido a que la referencia clínica analiza y valora poblaciones no expuestas, mientras que el monitoreo biológico valora poblaciones expuestas por su ocupación.

\section{LÍMITES DE ACCIÓN BIOLÓGICA (LAB)}

Los LAB son magnitudes del agente consideradas como aceptables bajo las condiciones de trabajo, es decir sopesan la captación del tóxico en el lugar de trabajo. Pueden ser valorados desde dos puntos de vista: 1) Valorando dosis-efecto y dosisrespuesta y 2) Por sus niveles ambientales en el área de labor.

En salud ocupacional, los LAB más usados son el índice de exposición biológica (BEI) de la Conferencia Americana para Higienistas Industriales Gubernamentales (ACGIH), el límite biológico de tolerancia en el trabajo (Biologische ArbeitsstoffToleranzwerte, BAT) para los químicos en general y el Expositionsequivalent für Krebserzeugende Arbeitsstoffe (EKA) para químicos carcinogénicos de la alemana DFG (Deutsche forschungs gemeinschaft) $\left({ }^{9,10}\right)$.

Muchos de los valores límites de acción biológica han sido derivados indirectamente de estudios preexistentes sobre el aire de los ambientes de trabajo, tal los valores límites umbral (TLVs) de la ACGIH o los alemanes Maximale Arbeitsplatz konzentrationen (MAK) y Technische Richt konzentrationen (TRK) de los carcinogénicos. La validez de los $\mathrm{LAB}$ varía de normas legales obligatorias a recomendaciones no obligatorias. Se especifica las magnitudes del metal en peso o masa, esta última unidad recomendada por la OMS (SI) $\left({ }^{9,10}\right)$.

Revisemos los BEIs de los metales pesados obtenidos con mayor frecuencia en la minero-metalurgia primaria del Perú y que deberían servirnos de guía cuando valoremos las exposiciones ocupacionales: 


\begin{abstract}
Aluminio
El aluminio (Al) ingresa por vía respiratoria. Cuando inhalamos humos de soldadura o partículas respirables de aluminio, éstas son retenidas y luego excretadas lentamente. El Al tiene efecto acumulativo lento en pulmones, riñones, hígado, bazo, músculos estriados y cerebro. En el tejido óseo de expuestos, la concentración fluctúa entre 18 y 29 ug/g, cinco veces más que para no expuestos, en quienes la concentración en suero es menor a $100 \mathrm{umol} / \mathrm{L}$ y en orina menor a $600 \mathrm{umol} / \mathrm{L}$.
\end{abstract}

Los BEIs ACGIH aceptados varían entre 200 y $400 \mathrm{umol} / \mathrm{L}$ en suero y 3 a $6 \mathrm{umol} / \mathrm{L}$ en orina $\left({ }^{10}\right)$.

\section{Antimonio}

La toxicocinética del antimonio y sus compuestos es poco conocida. Sin embargo, la inhalación ocupacional de óxidos produce acumulación en pulmones y su concentración en sangre y orina siempre es alta y se observa correlación entre concentración ambiental de antimonio y su concentración en orina $\left({ }^{8}\right)$.

El BEI en orina de expuestos es $35 \mathrm{ug} / \mathrm{g}$ creatinina $(32,5 \mathrm{umol} / \mathrm{mol})$ para un ambiente de $500 \mathrm{ug} / \mathrm{m}^{3}$ de antimonio $\left({ }^{10}\right)$.

\section{Arsénico}

Es un metaloide de mucha presencia en la metalurgia del cobre. Sus compuestos trivalentes tienen tendencia acumulativa en el organismo: en queratina de uñas, pelo, y piel.

Se excreta por orina, heces y células córneas de la piel. Al ingresar, el arsénico inorgánico es metilado, formando los ácidos dimetilarsínico y metilarsónico. En exposición ocupacional abundan los compuestos metilados, principalmente ácido dimetilarsínico que se excreta en la orina. Las proporciones de As trivalente, pentavalente y compuestos metilados en orina varían según la magnitud de exposición y la hora de toma de la muestra ${ }^{(11)}$.

Los peces y mariscos contienen grandes cantidades de arsénico orgánico (arsenobetaína). Al ingerirlos, son absorbidos por el tracto gastrointestinal y excretados en la orina. En no expuestos, el As inorgánico en orina es menor a $1 \mathrm{ug} / \mathrm{L}$.

La referencia DFG para As ambiental trivalente de $100 \mathrm{ug} / \mathrm{m}^{3}$ recomienda un EKA de $330 \mathrm{ug} / \mathrm{L}(4,4 \mathrm{umol} / \mathrm{L})$. Otras normas para exposiciones a $10 \mathrm{ug} / \mathrm{m}^{3}$, muestra pos tarea, va de 5 a $10 \mathrm{ug} / \mathrm{L}$ de orina $(70$ a 130 umol/L) $\left({ }^{9}\right)$.

El BEI ACGIH para un TLV de $10 \mathrm{ug} / \mathrm{m}^{3}$ es $50 \mathrm{ug} / \mathrm{g}$ creatinina $(75 \mathrm{umol} / \mathrm{mol})$ en orina e incluye compuestos solubles de As en muestra fin de tarea $\left({ }^{10}\right)$.

\section{Cadmio}

El cadmio ingresa al organismo vía respiratoria y gastrointestinal. En sangre, el $70 \%$ está en los hematíes. Se acumula en riñones e hígado y ya se ha determinado su carácter carcinógeno. Su monitorización biológica mide niveles en sangre, $\mathrm{Cd}-\mathrm{S}$ y en orina, Cd-U; ambos dan información complementaria. El Cd-S actual refleja exposición en meses precedentes; pero, medido después del cese de exposición refleja carga corporal. El Cd-U refleja exposición actual o reciente. En esta fase, refleja exposición reciente y carga corporal. Otros BEIs importantes son: lisozima, proteína ligada al retinol y beta 2 microglobulina $\left({ }^{12}\right)$.

El cadmio en orina en no fumadores no expuestos es menor a $15 \mathrm{nmol} / \mathrm{L}(1,5 \mathrm{ug} / \mathrm{L})$ y en sangre menor de $10 \mathrm{nmol} / \mathrm{L}(1 \mathrm{ug} / \mathrm{L})$. En fumadores no expuestos, el $\mathrm{Cd}-\mathrm{S}$ puede alcanzar $50 \mathrm{nmol} / \mathrm{L}$ y el $\mathrm{Cd}-\mathrm{U} 10 \mathrm{nmol} / \mathrm{L}$. 
El BEI ACGIH para el Cd-U es $10 \mathrm{ug} / \mathrm{g}$ creatinina (10 umol/mol) y para $\mathrm{Cd}-\mathrm{S}, 10$ $\mathrm{ug} / \mathrm{L}(90 \mathrm{nmol} / \mathrm{L})\left({ }^{9,10}\right)$.

\section{Cromo}

La absorción, distribución, y excreción del cromo (Cr) y sus compuestos son muy variables y dependen principalmente de las características físicas y de la solubilidad del compuesto. Algunos compuestos trivalentes se absorben rápidamente y su concentración urinaria es elevada. El análisis de cromo urinario $(\mathrm{Cr}-\mathrm{U})$ es usado para monitoreo biológico de expuestos. El Cr-U en no expuestos es menor a $10 \mathrm{nmol} / \mathrm{L}(0,5 \mathrm{ug} /$ L) $\left({ }^{13}\right)$.

El valor EKA para Cr-U en exposición a cromatos alcalinos es $40 \mathrm{ug} / \mathrm{g}$ creatinina (87 umol $/ \mathrm{mol}$ ) en muestra de orina fin de tarea.

El BEI ACGIH para Cr-U en expuestos a $\mathrm{Cr}$ VI es un incremento de $10 \mathrm{ug} / \mathrm{g}$ creatinina (22 umol/mol) durante una tarea, o $30 \mathrm{ug} / \mathrm{g}$ creatinina (65 umol/mol) en muestra fin de tarea o fin de semana de labor $\left({ }^{10}\right)$.

\section{Cobalto}

El cobalto (Co) metálico y sus sales se absorben rápidamente por vía respiratoria. Se acumula en hígado y se excreta por la orina en las primeras 24 horas de exposición y luego por heces. El Co-U en no expuestos es menor a $5 \mathrm{ug} / \mathrm{L}(85 \mathrm{nmol} / \mathrm{L})\left({ }^{13}\right)$.

El valor BAT para Co-U es de 60 a 300 ug/L (1 ó 5 umol/L), dependiendo del tipo de exposición y correspondiendo a un valor ambiental entre 100 y $500 \mathrm{ug} / \mathrm{m}^{3}$ en muestra de orina de fin de tarea $\left({ }^{9}\right)$.

\section{Manganeso}

El manganeso (Mn) ingresa al organismo por las vías respiratoria y gastrointestinal. En expuestos por largo tiempo, se halla síntomas asociados con déficit en pruebas neurosicológicas, pero sin relación con niveles actuales de $\mathrm{Mn}$ en sangre u orina. Sin embargo, se ha observado una marcada yuxtaposición de los valores de trabajadores expuestos y no expuestos $\left({ }^{13}\right)$.

Los BEIs Mn de la ACGIH en expuestos y no expuestos son similares en sangre, suero y orina y no deben ser mayores de 14,1 y $1 \mathrm{ug} / \mathrm{L}$, respectivamente $\left({ }^{10}\right)$.

\section{Mercurio}

La peligrosidad del mercurio metálico $(\mathrm{Hg})$ radica en que se evapora a temperatura ambiental, punto de evaporación $13^{\circ} \mathrm{C}$, y es absorbido fácilmente por la vía respiratoria. En pulmones, los vapores de $\mathrm{Hg}$ son oxidados a $\mathrm{Hg}$ II, ligándose inmediatamente a las proteínas. El mercurio se acumula en riñones, hígado, bazo y huesos. Otros estudios han informado de deterioro psicomotor en trabajadores con niveles de $\mathrm{Hg}-\mathrm{U}$ entre 250 y $500 \mathrm{nmol} / \mathrm{L}$ $\left({ }^{14}\right)$.

En general, la exposición a $\mathrm{Hg}$ es monitoreada con mercurio en sangre $(\mathrm{Hg}$ S) para exposiciones agudas o accidentales, y con mercurio en orina $(\mathrm{Hg}-\mathrm{U})$, para exposiciones ocupacionales a niveles ambientales constantes, no olvidando que la tasa de $\mathrm{Hg}$-S total puede tener grandes variaciones según la población en la que se mide. Así, es muy alto en aquellos grupos humanos con dieta basada en pescado ( $\mathrm{Hg}$ $\mathrm{S}$ mayor de $200 \mathrm{nmol} / \mathrm{L}$ (40 ug/L)), mientras que en poblaciones con dieta no basada en pescado, el $\mathrm{Hg}-\mathrm{S}$ está por debajo de 50 $\mathrm{nmol} / \mathrm{L}(10 \mathrm{ug} / \mathrm{L})$. Una cifra de referencia usada en salud general para el $\mathrm{Hg}-\mathrm{S}$ inorgánico es aproximadamente $125 \mathrm{nmol} /$ L. El Hg-U muestra marcada variación circadiana, que inclusive no puede compensarse totalmente corrigiéndola por densidad o excreción de creatinina. Por este hecho, la muestra llamada spot ha sido descartada y reemplazada por orina de 24 
horas $\left({ }^{15}\right)$. En no expuestos, el $\mathrm{Hg}-\mathrm{U}$ generalmente está por debajo de $50 \mathrm{nmol} / \mathrm{L}$ (50 ppb/ L o $10 \mathrm{ug} / \mathrm{L})$.

El BEI Hg de la ACGIH es $\mathrm{Hg}-\mathrm{U}$ menor a $40 \mathrm{ug} / \mathrm{L}, \mathrm{Hg}$ en sangre menor de $30 \mathrm{ug} / \mathrm{L}$ $\left({ }^{10}\right)$.

\section{Níquel}

Es un elemento demostradamente cancerígeno. Sus compuestos difieren notablemente en su toxicocinética. Así, el óxido metálico de níquel y sus sales no solubles son eliminadas por las mucosas respiratorias muy lentamente durante varias décadas.

$\mathrm{Su}$ valor de referencia es $1,3 \mathrm{umol} / \mathrm{L}$ posexposición y $1,0 \mathrm{umol} / \mathrm{L}$ al día siguiente. En general, siempre existe correlación cuando la exposición es a compuestos hidrosolubles de níquel; por eso, la concentración de Ni-U es un excelente indicador de exposición $\left({ }^{15}\right)$.

\section{Plomo}

En exposición ocupacional, el pulmón es su principal vía de ingreso y el $50 \%$ del $\mathrm{Pb}$ proveniente de esta vía se absorbe y distribuye a todo el organismo. Un $75 \%$ es excretado por los riñones. El indicador plomo en sangre ( $\mathrm{Pb}-\mathrm{S})$ es usado para el monitoreo biológico de exposición, pues refleja con fidelidad las concentraciones medias de plomo en los compartimentos de recambio $\left({ }^{16}\right)$.

Además, como BEIs se usa los derivados de la síntesis del núcleo HEM, tal la zincprotoporfirina (ZPP) en hematíes, la excreción urinaria de ácido delta aminolevulínico y la actividad de la dehidratasa del ácido delta amino-levulínico en hematíes $\left({ }^{17}\right)$.

Para plomo sanguíneo, la ACGIH adopta los BEIs normados por la OMS. La NIOSH da un BEI general de $40 \mathrm{ug} / 100 \mathrm{~mL}$ para varones y $30 \mathrm{ug} / 100 \mathrm{~mL}$ para mujeres en edad fértil y da pautas para el manejo de trabajadores expuestos $\left({ }^{10}\right)$.

\section{CONCLUSIONES}

El uso de biomarcadores en el monitoreo biológico del trabajador expuesto a tóxicos en metalurgia, ayuda en la mejora de las condiciones de trabajo, al reforzar la valoración del riesgo.

Conocer el manejo de los indicadores biológicos de exposición nos permite en salud ocupacional manejar eficaz y eficientemente la potencial agresión de los tóxicos, previniendo las enfermedades laborales.

La legislación peruana actual no establece valores de referencia para tóxicos industriales. Sin embargo, nos remite a las normas de la OIT o a referencias internacionales, que precisamente hemos revisado en este opúsculo.

\section{REFERENCIAS BIBLIOGRÁFICAS}

1. Lauwerys R, Hoet P, Industrial chemical exposure-guidelines for biological monitoring. $2^{\text {nd }} \mathrm{Ed}$. London: Lewis; 1993.

2. World Health Organization. Biological monitoring of chemical exposure in the workplace. Geneva: World Health Organization; 1996.

3. Christiani DC. Utilization of biomarker data for clinical and environmental intervention. Environ Health Perspect. 1996;104:921-5.

4. Coelho-André A L, O uso dos Biomarcadores na avaliacao da exposicao ocupacional a substancias químicas Rev Bras Med Trab, Belo Horizonte. 2003;1(2):124-32. Out-Dez. 2003.

5. Marquart H, Schafer S G, McClellan R, Eds. Toxicology. San Diego: Academic Press; 1999.

6. Manzo L, et al. Biochemical markers of neurotoxicity: a review of mechanistic studies and applications. Hum Ex Toxico. 1996;15(1):20-35.

7. Hemminki K. DNA Adducts in biomonitoring. J Occup Environ Med. 1995;37:44-9.

8. Aitio A, Riihimäki J, Liesvuoris J, Järvisalo J, Hernberg S. Biological monitoring. En: Zenz C, Editor. Occupational 
Medicine: Principles and Practical Applications. $2^{\text {nd }}$ Ed. Chicago: Mosby; 1988.

9. ACGIH, TLVs, BEIs. Threshold limits values for chemical substances and physical agents. Biological exposure indices. Cincinnati: ACGIH; 2003.

10. Deutsche Forschungsgemeinschaft: MAK und BAT -WerteListe 1992. Weinheim, BRD: VHC Verlagsgesellschaft; 1992.

11. Ramírez AV, Castillo EC. Arsenicismo ocupacional en metalurgia extractiva primaria. Salud, Trabajo y Medio Ambiente. Montevideo: 1998. p. 11.

12. Ramírez AV. Poluçâo ambiental por Cd: efeitos en um grupo humano nao exposto ocupacionalmente. Rev Bras Saúde Ocupacional. 1985;50(13).

13. Ellemhorn MJ. Medical Toxicology. $2^{\text {nd }}$ Edition. Baltimore: Williams Wilkins; 1996.

14. Levy BS, Wegman DH, Eds. Occupational Health. Boston: Little, Brown and Co.; 1988.

15. Friberg L, Nordberg GF, Vouk VB, Editors. Handbook of toxicology of metals. Amsterdam: Elsevier. 1979.
16. Ramírez AV. El cuadro clínico de la intoxicación ocupacional por plomo. An Fac Med Lima. 2005;66(1):57-70.

17. Ramírez AV, Tapia G. Saturnismo en la industria metalúrgica de la altura. Bol Soc Peruana Medicina Interna. 1997;10:1538.

Manuscrito recibido el 10 de diciembre de 2005 y aceptado para publicación el 06 febrero de 2006.

Correspondencia: Dr. Augusto V Ramírez.

Las Mandarinas 210 Apto. 306

Lima 12, Perú

Correo: augustovram@yahoo.es 